\title{
AC-ELECTROKINETICS BASED TOOLS IN NANOENGINEERING AND MOLECULAR ELECTRONICS
}

\author{
A Ramírez, A. Zehe \& R. Durán \\ Benemérita Universidad Autónoma de Puebla, \\ Facultad de Cs. de la Electrónica, Dept. Posgrado, \\ Apdo. Post. \# 1505, 72000 Puebla, Pue., México. \\ aramirs@siu.buap.mx
}

Received: May 28 $8^{\text {th }}, 2003$. Accepted: April 21 ${ }^{\text {st }}, 2005$

\begin{abstract}
Silicon-based microelectronics has been following the integration prognosis of MOORE's Law during the past decades and possibly will do so for another decade or two. Physical, technological and also financial limits in the foreseeable future will slow down the continued expansion of this branch of microelectronics and instead will force a new technological approach based on molecular-scale electronics (MOLETRONICS). New tools are needed to allow molecular device manufacturing and nanoscale engineering with high precision and productivity. One group of methods with the potential for use in such a manufacturing process is based on a.c. electrokinetics effects, which are described and discussed in this paper.
\end{abstract}

\section{RESUMEN}

La microelectrónica en base a silicio ha seguido la prognosis de integración según la ley de MOORE durante las décadas pasadas, y posiblemente continuará así por otra década más. Límites físicos, tecnológicos y también financieros en el futuro cercano reducirán la razón de expansión de esta rama microelectrónica, y en su lugar generará una aproximación tecnológica novedosa, que se basa sobre la electrónica a escala molecular (MOLETRÓNICA). Se requieren nuevas herramientas, que permitan la fabricación de dispositivos moleculares y la ingeniería a escala nanométrica con alta precisión y productividad. Un grupo de métodos con el potencial de ser usado en tal proceso de fabricación se basa en efectos electrocinéticos en campos eléctricos alternos, que en el presente trabajo se describe y discute.

KEYWORDS: Embedded Electrokinetics, Dielectrophoresis, Electrorotation, Dielectric Polarization, Nanoengineering.

\section{INTRODUCTION}

Nanotechnology comprises the ability to manipulate atoms and molecules on an individual level. It will lead to extremely fast but inexpensive computers, smart fabrics and microscopical medical devices [1-3]. One of the goals of nanotechnology is to build nano-scale machines that operate at the atomic and molecular level [4 \& 5]. Thus surges the indispensable need for molecular design tools, which would allow the assembly by manipulating and positioning basic building blocks [6-10].

Current technological achievements in semiconductor based microelectronics, and particularly in the reproduction of fine structures on the nanometer scale, provide for a 'top-down' approach of structure building on the nanometer scale. While semiconductor based microelectronics encounters physical limits for a continued reduction in 
geometrical device features in the foreseeable future, molecular-scale electronics (Moletronics) is emerging to provide for an even more powerful advancement of computer technology [11-13]. The majority of molecules are Intrinsically small and thus already of the size scale, that present CMOS technology is driving toward (CMOScomplementary metal oxide semiconductor). Here again appears the need for molecular design tools, possibly in combination with modern synthetic chemistry techniques allowing atomic control over a wide variety of molecular structures, as an obvious prerequisite. The possibility to synthesize organic molecules with desired structure and functionality in conjunction with sophisticated thin-film deposition technologles enables now the production of electrically and biologically active devices on the nanometer scale. The deposition of an organic film can be carried out by varlous techniques $[15 \& 16]$, such as thermal evaporation, electrodeposition, molecular-beam epitaxy, adsorption from solution, and by the Langmuir-Blodgett technique. The latter allows a precise control of the molecular monolayer thickness and arrangement on almost any kind of solid substrate. However, even though many ideas for practical application exist, these films are still mostly used as model systems or multilaver coatings in biomembrane research [17 \& 18].

The incorporation of blological materials into electronic concepts, that is, the formation of nanobiosystems, follows fundamental principles that blology uses in order to produce nanodevices. Contrary to the highly contaminating semiconductor technology, these artificlal structures are synthesized at room temperature using benign aqueous solvents in a 'bottom-up' approach. Self-assembly of structures and devices for basic biological or chemical building blocks is considered to be the ideal approach, through which nanotechnology will ultimately be implemented, but until this goal is reached the manipulation of components with much larger devices will be a necessary step on this way [14].

Electrokinetic effects, which occur due to the interaction of induced dipoles with electric fields, have been used for many years for the manipulation and analysis of biological cells and particles in the micrometer-size scale. A variety of motions can be carried out by changing the nature of the dynamic electric field, including attraction, repulsion and rotation [19-21]. The equipment used is simple, and can be constructed with existing techniques of semiconductor manufacturing, starting with photolithography and getting finally to electron-beam lithography. Electrodes were fabricated, which enabled the manipulation of macromolecules as small as $9 \mathrm{kDa}(1$ Dalton corresponds to one atomic mass unit), using attractive or repulsive a.c. electrokinetic forces [22]. Simple particles, such as viruses, have been trapped in contactless potential energy wells, and nanosized particle populations of latex spheres and tobacco mosaic virus have been moved and separated [23]. A wealth of further experimental results exists, which allows the prognosis, that such a technology will ultimately have applications for the manipulation of simple molecules [24-28].

In the present paper we are going to discuss some physical aspects related to a.c. electrokinetic forces in dielectric objects with geometries deviating from common ellipsoidal shapes. Electrokinetic effects are involved in e.g. electroorientation, dielectrophoresis, electrorotation and electrodeformation of tiny solid particles with a given body shape by virtue of interactions between the local electric field vector and the induced dielectric polarization vector (Fig. 1). The latter requires particular consideration, If the particle shape cannot be approximated by an ellipsoidal body. As the calculation of a.c. electrokinetic force effects requires the precise knowledge of the dielectric polarization, we will deduce in a first step the induced dipole moment of a dielectric body of general shape, which is exposed to an external electric field $E(\vec{r})$. Dielectrophoresis and electrorotation forces will then be calculated for dielectric objects of cylindrical shape. Such objects are represented e.g. by the tobacco mosaic virus (TMV) of about 100 to $280 \mathrm{~nm}$ in length and $18 \mathrm{~nm}$ in diameter, or by so-called 'rouleaux' of human erythrocyte cell aggregates of about $7 \mu \mathrm{m}$ in diameter and varlable 'cylinder' length, depending on the number of stacked enthrocytes (helght of any about 2.2 $\mu \mathrm{m})$. 
a

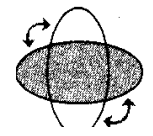

b)

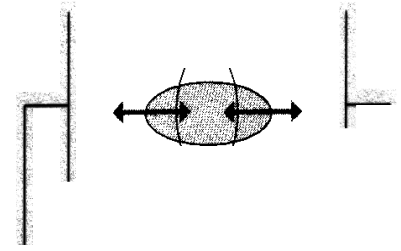

(d)

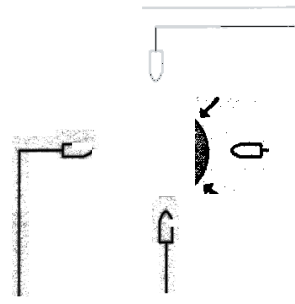

C)

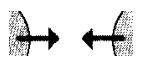

(e)

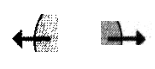

Figure 1. Schematic presentation of dielectric effect in alternating homogeneous electric fields

(a) Orientation of a non-spherical cell; (b) Deformation (elongation, compression);

(c) Attraction between several particles; (d) Entrapment of cells or particles;

(e) dielectrophoresis; (f) rotation of a particle

\section{POLARIZATION IN CYLINDRICAL OBIECTS}

Only in very few cases can the electric field $E(\vec{r})$ in the presence of matter with dielectric constant $\varepsilon$ be calculated. In such cases an object of special shape is usually brought into a special electric field $E_{0}(\vec{r})$. With an intelligent guess is then shown that the boundary conditions of the flelds on the surface of the dielectric material are fulfilled [29].

Dielectric models, applied in diverse research areas, consider the structural properties of matter by assuming spherical or general ellipsoidal geometries. This is widely used, e.g., in the case of the interaction of a.c. electric fields with colloidal particles and biological cells. A variety of methods, based on impedance measurements and on different force effects are employed for the dielectric characterization of single objects [30]. The general Laplace solution for the polarization of single-shell ellipsoids, a standard model of biological cells [31], was also derived for the meteorological problem of dust particles covered by a water layer [32]. In the Laplace model, a homogeneous ellipsoid always exhibits a constant internal local field. Integrating over this field leads to the induced dipole moment and thus to analytical expressions related to force actions on the particles.

Already in such important cases as a cube or a cylinder it is difficult to calculate the depolarization factors without assuming a spheroid as substituting body shape. But even then the best shape of it has to be chosen, and the design of the next approximation step has to be done, and not straightforward. Driven by the growing interest and impact of physical contributions to life sciences and nanotechnologies, complex geometries, such as rods and cylinders, need to be considered. Characterized by the unavailability of analytical solutions for the field distribution within such dielectric 
bodies, finite element numerical techniques have been developed recently [33], though with the compromise of claiming consideráble computer resources.

\subsection{Depolarization Field Calculation}

The problem, which is treated here, can be formulated as follows: The internal field $E_{i}(\vec{r})$ in a dielectric object generates a polarization $P(\vec{r})$. This polarization induces on the surface element $\Delta F$ of the dielectric body a polarization charge $\Delta q=\sigma_{p o l} \cdot \Delta F=P \cdot \Delta F$, which by virtue of the Coulomb law, together with the unperturbed field $E_{0}(\vec{r})$, generates finally the depolarization field, such that

$$
E_{i}\left(\vec{r}_{1}\right)=\vec{E}_{0}\left(\vec{r}_{1}\right)-\oiint \frac{\vec{r}_{12}}{4 \pi \varepsilon_{0} r_{12}^{3}}\left(\vec{P}\left(\vec{r}_{2}\right) \cdot \Delta \vec{F}_{2}\right)
$$

The integration is carried out over the surface of the dielectric body; $\Delta F$ points outward, and $\vec{r}_{12}$ combines the origin at $r_{1}$ with the integration element at $r_{2}$ as shown in Fig. 2. The relation between $E_{0}(\boldsymbol{r})$ and $E_{i}(\boldsymbol{r})$ is supposed to be lineal

$$
\vec{P}(\vec{r})=\varepsilon_{0}(\varepsilon-1) E_{i}(\vec{r})=\varepsilon_{0}(\varepsilon-1) \alpha(\vec{r}) E_{0}(\vec{r})
$$

In general, $\alpha(\vec{r})$ is a tensor, as the directions of $E_{i}$ and $E_{0}$ are not necessarily parallel. It further depends on the coordinates inside the sample due to the locally different action of the polarization charges. In order to calculate $\vec{P}(\vec{r})$ or $E_{i}(\vec{r})$ from equations $(1,2)$, we make the assumption, that $\alpha$ does not depends from $\vec{r}$, which of course is exactly fulfilled only in homogeneous ellipsoids. Here it is an approximation, which allows us to get viable solutions.

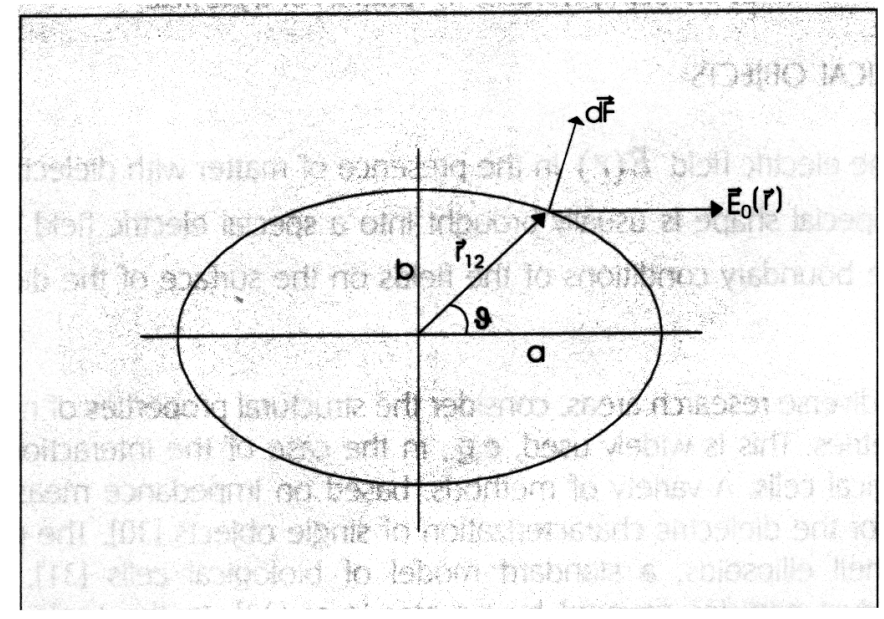

Figure 2. Prolate ellipsoid and symbols as used in the calculus

The polarization, established inside the dielectric, is due to the displacement of electrical charges enforced by the field $E_{0}(r)$. Surface charges are built up and counteract the complete displacement corresponding to the field $E_{0}(\vec{r})$. We will suppose here, that the whole set of charges experiences an identical displacement, which means, that $\alpha=$ constant. We further suppose, that the polarization vector $P$ points more or less into the direction of 
$E_{0}(\vec{r})$, l.e., we will consider the projection of the field, generated by the polarization charges, on the direction of $E_{0}(\vec{r}): \vec{P}\left(\vec{r}_{1}\right)=\varepsilon_{0}(\varepsilon-1) \alpha_{1} E_{0}\left(\vec{r}_{1}\right)$

$$
\alpha_{1}=\left\{1+\frac{\varepsilon-1}{4 \pi} \oiint \frac{\left(E_{0}\left(\vec{r}_{2}\right) \cdot d F_{2}\right)}{r_{12}^{3}} \cdot \frac{\left(E_{0}\left(\vec{r}_{1}\right) \cdot r_{12}\right)}{E_{0}^{2}\left(\vec{r}_{1}\right)}\right\}^{-1}
$$

This value $\alpha_{1}$ allows to consider a first approximation of the polarization $\vec{P}_{1}$, which on the surface of the dielectric object generates charges, and thus an additional field inside the dielectric. The problem would be completely solved, If the total field at any place already fulfills the condition $E_{i}=P_{1} / \varepsilon_{0}(\varepsilon-1)$ but in general, the polarization $\vec{P}_{1}$ of the first approximation step will not be sufficient to describe the real situation, and a field $E_{1}(\vec{r})$ keeps acting on the dielectric body with the effect of an additional polarization $\vec{P}_{2}(\vec{r})$

$$
\vec{E}_{1}(\vec{r})=\vec{E}_{0}(\vec{r})-\iint \frac{\vec{r}_{12}}{4 \pi \varepsilon_{0} r_{12}^{3}}\left(\vec{P}_{2}\left(\vec{r}_{2}\right) \cdot d \vec{F}_{2}\right)-\vec{P}_{1}\left(\vec{r}_{1}\right) / \varepsilon_{0}(\varepsilon-1)
$$

$\vec{P}_{2}(\vec{r})$ can now be calculated with $E_{1}(\vec{r})$ in the same way as $\vec{P}_{1}(\vec{r})$ was calculated with $E_{0}(\vec{r})$

The number of approximation steps needed to achieve the best result depends on the complexity of the shape of the dielectric body, as well as the allowed error of the result.

\subsection{The Depolarization Field in a Short Dielectric Cylinder}

Exact solutions are known for the sphere, the infinitesimal thin wire and the infinitesimal extended disk. When our approach is applied here, already the first approximation step gives the exact solution, as it should be, when $\alpha(\vec{r})=$ $\alpha_{1}=$ constant.

The polarization of a prolate spheroid (Fig. 2) results with equation 2 in

$$
\left.\alpha=-(\varepsilon-1) q^{2}\left(+\frac{\sqrt{q^{2}+1}}{2} \cdot \ln \frac{\sqrt{q^{2}+1}-1}{\sqrt{q^{2}+1+1}}\right)\right\}
$$

where $q^{2}=b^{2} /\left(a^{2}-b^{2}\right)$.

For an oblate ellipsoid one gets

$$
\alpha=\left\{1-(\kappa-1)\left(q^{2}+1\right)\left(q \arctan \frac{1}{q}-1\right)\right\}^{-}
$$

and consequently with $\mathrm{q} \rightarrow \infty$ (or $\mathrm{a}=\mathrm{b}$ ) we have for the sphere-shaped dielectric $\alpha=3 /(\varepsilon+2)$, and thus the known result for the polarization of a sphere 


$$
P=3 \varepsilon_{0} \frac{\varepsilon-1}{\varepsilon+2} \cdot E_{0}
$$

Not so straightforward is the situation in the case of a cylinder in a homogeneous electric fleld $E_{0}(\vec{r})$ (see fig. 3 ).

We get

$$
\iint \frac{\vec{E}_{0}\left(\vec{r}_{2}\right) \cdot d \vec{F}_{2}}{\vec{P}_{12}^{3}} \frac{E_{0}\left(\vec{r}_{1}\right) \cdot \vec{r}_{12}}{E_{0}^{2}\left(\vec{r}_{1}\right)}==2 \int_{0}^{R} \frac{2 \pi a d a \cdot L}{\left(a^{2}+L^{2}\right)^{3 / 2}}=\int_{L}^{\left(R^{2}+L^{2}\right)^{1 / 2}} \frac{r d r}{3} 4 \pi L=4 \pi\left[1-\left(1+R^{2} / L^{2}\right)^{-1 / 2}\right],
$$

$$
P_{1}=\frac{\varepsilon_{0}(\varepsilon-1) E_{0}}{1+(\varepsilon-1)\left(1-\left(1+R^{2} / L^{2}\right)^{-1 / 2}\right)}
$$

Such an homogeneous polarization is only the first approximation. Due to the choice of the origin at $\mathbf{Z}=0$, the $\mathbf{P}$ generated field will be too weak in the transversal plane at $z=0$, but along the $z$-axis, at the limiting faces of the cylinder, it is too strong. A field $E_{1}(\vec{r})$ remains as given in Equation (4), which delivers the depolarization at the cylinder top and bottom faces in a second approximation step.

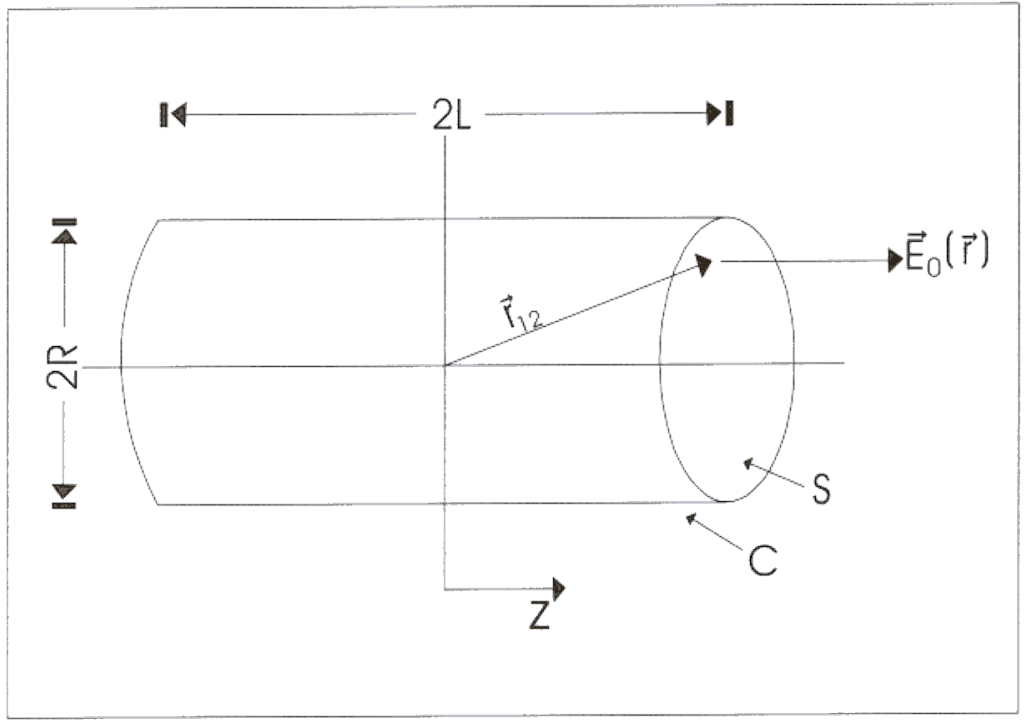

Flgure 3. Dielectric cylinder of length $2 L$ and Diameter $2 R$ s and $C$ are top surface and rim, respectively, where polarization charges accumulate

The integrations, involved in this step, are quite tedious and will not be carried out here. For more details see [42].

The dipole moment of the cylinder in a second approximation yields then

$$
P=\varepsilon_{0}\left(\varepsilon_{p}^{*}-\varepsilon_{M}^{*}\right) E_{0} \cdot \alpha
$$




$$
\begin{gathered}
\alpha=\left\{\varepsilon_{m}^{*}+\left(\varepsilon_{p}^{*}-\varepsilon_{m}^{*}\right)\left[1-\left(1+R^{2} / L^{2}\right)^{-1 / 2}\right]\right\}^{-1} \\
\left\{1+\frac{1 / 2-\left(1+R^{2} / L^{2}\right)^{-1 / 2}-(1 / 2)\left(1+8 L^{2} / R^{2}\right)^{-1}+(1 / 2 L)\left(R^{2}+2 L^{2}\right) \cdot\left(R^{2}+4 L^{2}\right)^{-1 / 2}-(1 / 2 L) R}{\left(\varepsilon_{p}^{*}-\varepsilon_{m}^{*}\right)^{-1}+1-7 / 16\left(1+11 R^{2} / 56 L^{2}\right)}\right\}
\end{gathered}
$$

Here we have included already an experimental condition of electrokinetic force manipulation, where the cylindershaped body of length $L$ and radius $R$ possesses a complex dielectric constant $\varepsilon_{p}^{*}$ and is embedded in a liquid suspending medium of complex dielectric constant $\varepsilon_{m}^{*}$.

\section{ELECTROKINETIC EFFECTS}

\subsection{Dielectrophoresis (DEP)}

Dielectrophoretic forces are caused by the interaction of non uniform electric fields with dielectric objects, which are suspended and free to move in a conductive medium, as shown in Fig. 1.

In inhomogeneous a.c. fields, the time averaged force $\langle F\rangle$, which is acting on a homogeneous dielectric particle, can be expressed by

$$
<\vec{F}>=\frac{1}{2} \operatorname{Re}\left\{\vec{m} \cdot \nabla E^{*}\right\}=\frac{1}{2} \cdot V \cdot \operatorname{Re}\left\{P \nabla E^{*}\right.
$$

where the induced dipole moment $\bar{m}=V \cdot P . \nabla E^{*}$ is the gradient of the complex conjugate of the external field, and Re denotes the real part of the scalar product. The induced dipole moment $\bar{m}$ is proportlonal to the particle volume $V$, the acting external electric field $\mathrm{E}=\mathrm{E}_{0} \mathrm{e}^{\mathrm{j} \omega \mathrm{t}}$ of circular frequency $\omega$, and the complex permittlvity $\varepsilon_{0} \varepsilon_{m}^{*}$ of the medium surrounding the dielectric object.

The time-averaged force acting on a homogeneous ellipsoldal particle is given by

$$
<F_{D E P}>=\varepsilon_{0} \varepsilon_{m} \cdot V \cdot \operatorname{Re}\{K(\omega)\} \nabla\left|E_{r m s}\right|^{2}
$$

with

$$
K(\omega)=\alpha \cdot\left(\varepsilon_{p}^{*}-\varepsilon_{m}^{*}\right),
$$

and by use of Equation (7)

$$
K(\omega)_{x}=\frac{\varepsilon_{p}^{*}-\varepsilon_{m}^{*}}{\varepsilon_{m}^{*}+\left(\varepsilon_{p}^{*}-\varepsilon_{m}^{*}\right) \cdot n_{x}},
$$


the component in x-direction of the Clausius-Mossotti factor $K(\omega) . \varepsilon_{p}^{*}$ is the complex permittivity of the particle, and $n_{x}$ is the Lorentz depolarization factor in x-direction, parallel to the external field. The Clausius-Mossottl factor is a measure of the effective polarizability of the particle, and depends for $n_{x}$ strongly on the geometrical shape of the ellipsoidal object. With $\varepsilon$ the permittivity and $\sigma$ the electrical conductivity of any dielectric medlum, the complex permittivity is defined as

$$
\varepsilon^{*}=\varepsilon-j\left(\sigma / \varepsilon_{0} \omega\right)
$$

being $\mathrm{j}$ the imaginary unit $(-1)^{1 / 2}$

Consequently, the Clausius-Mossotti factor depends on the frequency of the applied field, besides the dielectric properties of particle and medium. When only frequency dependencies are the objective of the study, it is sufficient to consider $K(\omega)$ as the only frequency-dependent part of the induced dipole moment. Variations of this factor give rise to the dielectrophoretic force described in (9), which is unique to a special particle type. This concerns not only intrinsic dielectric properties, but also the geometrical shape via the depolarizatlon factors and the size via the volume contained in the induced dipole moment.

Shape and size variation of the particles affect $K(\omega)$. and $V$, which leads to readily achievable dielectrophoretic separation protocols. The design and geometry of the microelectrodes used to generate and control the non-uniform electric field is also an important factor to be considered. The force $\left\langle F_{D E P}\right\rangle$ is toward the high electric field, and the particles collect at the point electrode edges, if $\operatorname{Re}\{K(\omega)\}>0$, on the contrary the force is in direction of the decreasing fleld, if $\operatorname{Re}\{K(w)\}$ is negative.

From expression (10) follow two special cases of practical importance: on one hand sphere-shaped particles with $\mathrm{n}_{\mathrm{x}}=$ $\mathrm{n}_{\mathrm{y}}=\mathrm{n}_{\mathrm{z}}=1 / 3$, yielding

$$
\left\langle F_{D E P}>_{\text {sphere }}=2 \pi R^{3} \varepsilon_{0} \varepsilon_{m}^{*} \operatorname{Re}\left\{\begin{array}{c}
\varepsilon_{p}^{*}-\varepsilon_{m}^{*} \\
\varepsilon_{p}^{*}+2 \varepsilon_{m}^{*}
\end{array}\right\} \nabla E^{2}\right.
$$

and on the other hand long cylinder-shaped particles with $\mathrm{n}_{\mathrm{x}}=0,\left(\mathrm{n}_{\mathrm{v}}=\mathrm{n}_{\mathrm{z}}=0.5\right)$

$$
<F_{D E P}>_{\text {needle }}=\frac{\pi R^{2} L}{3} \varepsilon_{0} \varepsilon_{m}^{*} \operatorname{Re}\left\{\frac{\varepsilon_{p}^{*}-\varepsilon_{m}^{*}}{\varepsilon_{m}^{*}}\right\} \nabla|E|
$$

where $L$ is the length, and $R$ is the radius of the long cylinder. It is clear, that the applicability of expressions $(5,6)$ has to be verified with respect to the particle shape in any practical approach.

Figure 4 represents a case, where a short cylinder of length $2 L$ and diameter $2 R$ is considered. The cylinder length is increased by discrete discs to form a 'coin stack' (insertion in Fig. 4). Let $s$ be the stack number, defined by $s=L / R$, then the shortest cylinder is given by $s=1$, and increasing stack numbers corresponds to increasing cylinder lengths. Large values of $s$ mean the border case of a large cylinder, given in equation (13). It is not surprising to see in Fig. 4 , that the positive dielectrophoretic force grows with $s$, which is mainly a consequence of the growing volume of the cylinder. On the other hand, display cylinders of different length (different stack numbers) crossing points on the frequency scale, where the direction of the dielectrophoretic force turns around. Between two populations of, say, short and long cylinders, a frequency range of the electric field is manifested, where short cylinders are attracted, while long cylinders feel repulsion from one side of both electrical contacts. 


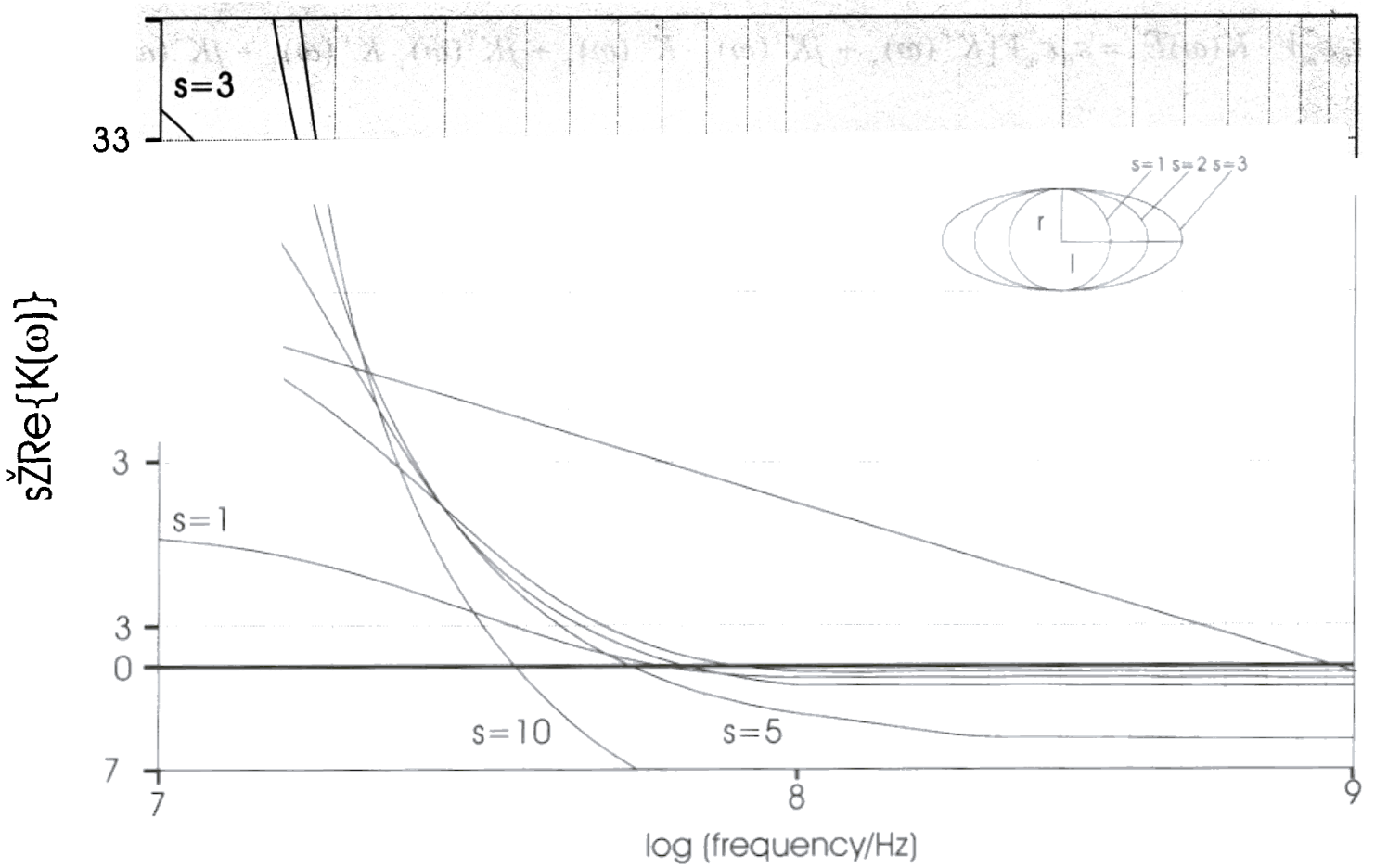

Figure 4. A plot of the real part of Clausius-Mossotti factor against a.c. field frequency,

for short cylinders of diameter $2 R$ and different length $2 L^{\prime}$.

\subsection{Flectronotation (ER)}

A circularly polarized rotating electrical field induces a circulating dipole moment. Due to ever present dispersion processes in the exposed sample, the relaxation time of the dipole, a spatial shift between the external field vector and the induced dipole moment, happens and the dipole will lag behind the field. The interaction of the out-of-phase part of this dipole moment with the electrical field causes a torque in the body and forces it to rotate asynchronously with the field. The rotation can even be against the direction of rotation of the field, depending on whether the lag is more than $180^{\circ}$.

The time-averaged rotational torque in this conditions exerted on a particle is given by the vector product of induced dipole moment and conjugate field

$$
<T>=\frac{1}{2} \cdot V \cdot \operatorname{Im}\left[P x E^{*}\right]
$$

The induced dipole moment $m=V \cdot P$ is proportional to the external field $\mathrm{E}$, the suspending medium permittivit $\varepsilon_{m}^{*}$ and the volume $V$ of the object. Let the principal axes of an (ellipsoidal) object be oriented in parallel to the vectors of the base system, then $m$ is given by

${ }^{1}$ The stack-number $s=L / R$ takes values $s=1$ for the shortest cylinder, and $s=2,3, \ldots$ for cylinders of increasing length by the discrete measure s.L. Calculations are based on erythrocyte aggregates ('coin stacks'), using conductivities $\sigma$ of the suspending medium and the biological cell of $\sigma_{\mathrm{m}}=0.01 \mathrm{~S} / \mathrm{m} ; \sigma_{\mathrm{p}}=0.5 \mathrm{~S} / \mathrm{m}$; and permittivities $\varepsilon_{\mathrm{m}}=80, \varepsilon_{\mathrm{p}}=50$; $2 \mathrm{R}=7.5 \mu \mathrm{m} . \mathrm{s}=1$ corresponds to a side by side aggregate of 3 erythrocytes, $s=2$ to 6 erythrocytes, etc. 


$$
m=\varepsilon_{0} \varepsilon_{m}^{*} V \cdot K(\omega) \vec{E},=\varepsilon_{0} \varepsilon_{m}^{*} V\left[K^{r}(\omega)_{x}+j K^{i}(\omega)_{x} \cdot K^{r}(\omega)_{y}+j K^{i}(\omega)_{y} K^{r}(\omega)_{z}+j K^{i}(\omega)_{z}\right] \cdot E,
$$

where I, r - imaginary and real part, respectively.

With the external a.c. field written $E=E_{0} \cdot \exp (j \omega t)$, and supposing its components $E_{x}, E_{y}, E_{z}$ being parallel to the same orthonormal base system of the ellipsoid, it follows from expression (14)

$$
<T>=\frac{1}{2} \operatorname{Im}\left(\begin{array}{l}
m_{y} E_{z}^{*}-m_{z} E_{y}^{*} \\
m_{z} E_{x}^{*}-m_{x} E_{z}^{*} \\
m_{x} E_{v}^{*}-m_{v} E_{x}^{*}
\end{array}\right.
$$

An electric field circulating with constant amplitude in the $x-y$ plane can be written as

$$
E_{y}=j E_{x} ; E_{y}^{*}=-j E_{x}^{*}
$$

For $\mathrm{E}_{\mathrm{z}}$ being zero, and as established in practical cases, that one of the principal axes of the ellipsold is always perpendicular to the field plane, the $k$-th component $(k=x, y)$ of the acting torque results in

$$
<\mathrm{T}>_{k}=1 / 2 \varepsilon_{0} \varepsilon_{m}^{*} V E_{0}^{2} K^{i}(\omega)_{x}+K^{i}(\omega)_{y} k
$$

In analogy to the dielectrophoresis case, the Clausius-Mossottl factor in k-direction is given by

$$
K(\omega)_{k}=\alpha_{k} \cdot\left(\varepsilon_{p}^{*}-\varepsilon_{m}^{*}\right)
$$

Friction of the dielectric objects within the liquid medium might affect the mobility in both electrorotation and dielectrophoresis. Ignoring buoyancy forces and Brownian motion, the equation of motion can be written as

$$
m \frac{d v}{d t}=F_{E k}-F_{d r a g}
$$

where $F_{E k}$ is either the electrorotation force or the dielectrophoretic force. The instantaneous velocity $v$ is proportional to the instantaneous electrokinetic force, provided low Reynolds numbers (small particles), that is

$$
\mathrm{U}=\mathrm{F}_{\mathrm{Ek}} / \mathrm{f}
$$

and substituting for (taken here as) $F_{E R}$

$$
\mathrm{v}=\frac{V \cdot \varepsilon_{0} \varepsilon_{m}^{*} \operatorname{Im}[K(\omega)] E_{0}^{2}}{2 f}
$$

for a spherical particle is $f=6 \pi R \eta$, and so its mobility depends on the surface area. 
There are significant differences between the electrorotation force and the dielectrophoretic force accordin equations (9) and (14). While the former contains the square of the electric field, the latter depends on the gradient of the square of the electric field. More importantly, the torque depends on the imaginary part of the Clausius-Mossotti factor, while the electrophoretic force goes with the real part. The importance of this difference rests in the fact, that a particle may experience both dielectrophoretic force and torque simultaneously.

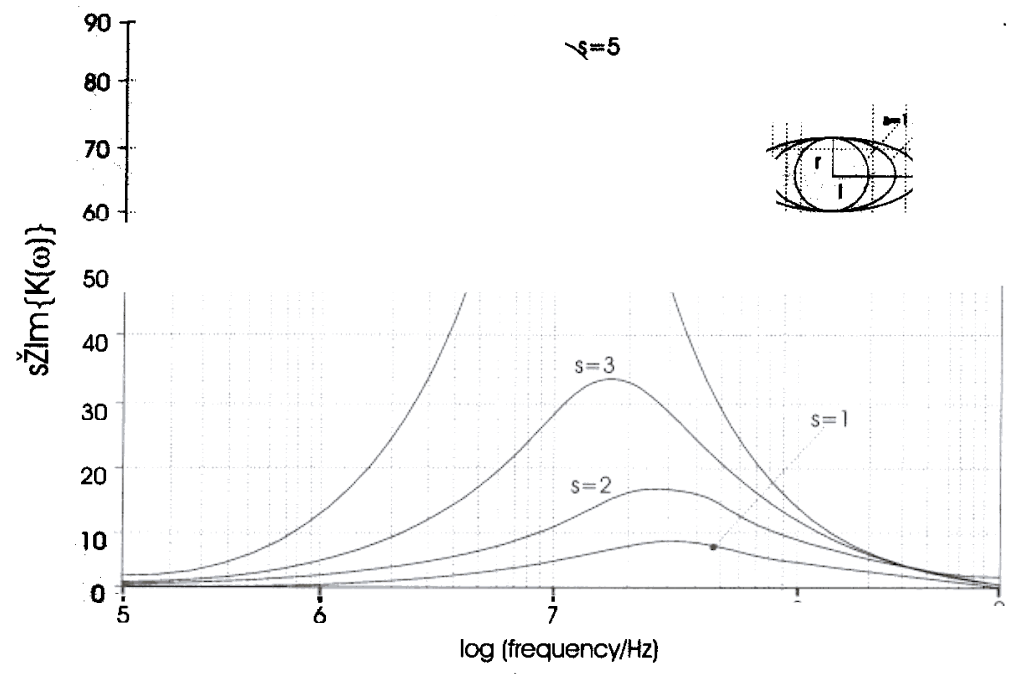

a)

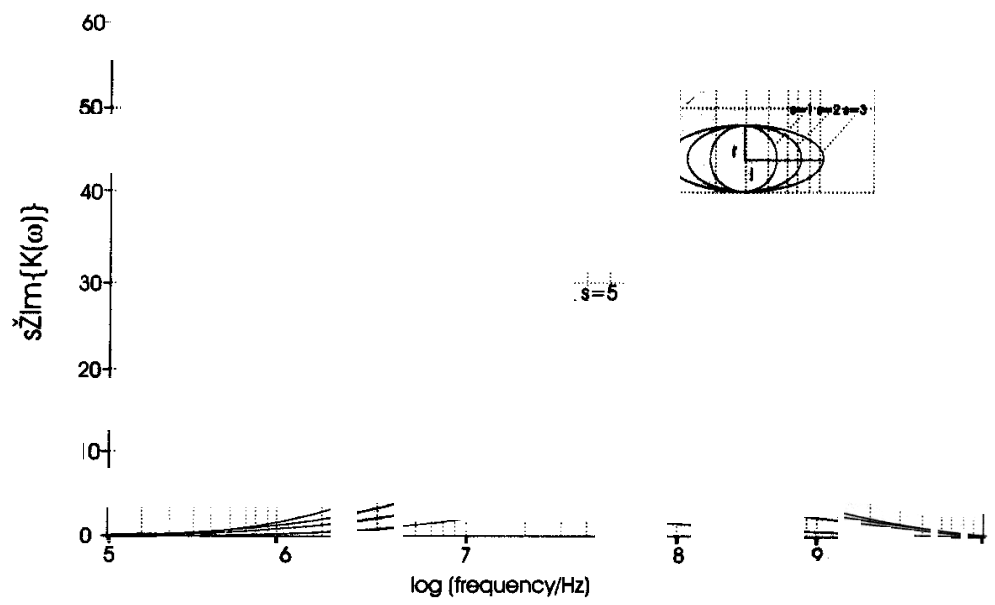

b)

Figure 5. Electrorotation spectra. ${ }^{2}$

In Fig. 5 we show the dependence of the torque (as being proportional to the imaginary part of the Clausius-Mossotti factor) of a cylindrical particle with different cylinder axis lengths, on a frequency of the electric field. The factor $s=$ $L / R$ is used here as explained in Flg. 4. While short cylinders (small $s$ ) display a maximum of the torque at high field frequencles, the longer the cylinder, this frequency maximum moves toward small values. The effect of different electrical conductivities of the suspending medium is seen in both, absolute torque and frequency of the torque maximum. The more pronounced effect occurs in the suspending medium of lower electrical conductlvity (FIg. $5 \mathrm{a}$ ).

${ }^{2}$ The torque is being proportional to $\operatorname{Im}[\mathrm{K}(\omega)]$ vs. the frequency $\omega$ of the electric rotation field. The stack number $\mathrm{s}$ and applied parameters are explained in Fig. 4. The difference between figs. (a) and (b) is caused by different values for the medium conductivity $(\mathrm{a}) \sigma_{\mathrm{m}}=0.001 \mathrm{~S} / \mathrm{m}$; (b) $\sigma_{\mathrm{m}}=0.01 \mathrm{~S} / \mathrm{m}$ with otherwise identical values 
A linear analogue of electrorotation is produced, when electrodes are laid out as tracks instead of being arranged in a circle. If the relationship of the electric field phases remains (that is, each following electrode is advanced in phase by $90^{\circ}$ with respect to the last), an electric field wave $(\lambda)$ moves along the electrodes. The result is the induction of a force rather than a torque with the particles moving along the electrodes. This effect is known as traveling-wave dielectrophoresis (TWD) and can be calculated with

$$
F_{T \omega D}=\frac{<T>}{\lambda}
$$

Particularly this effect has been widely studieds, including the demonstration of practical devices for biotechnological applications and electrostatic pumping.

\subsection{Electrode Configuration}

Electrode arrays as shown in Fig. 1 employ patterned electrodes fabricated of gold or some other conductor deposited on glass or on silicon wafers. Indeed there exists a relatively large number of different configurations, depending on the degree of field inhomogenelties required $[34,35]$. Very common is a quadruple design as show in Fig. 6, which allows both dielectrophoresis and electrorotation experiments. Advances in fabrication technology (electron-beam lithography) will allow the manufacture of electrodes with feature sizes of less than $100 \mathrm{~nm}$. Electric fields as high as $10 \mathrm{MVm}^{-1}$ can be generated by applying 10 volts across an one-micrometer inter-electrode gap. The manipulation of single nanometer-scale particles should then be possible.

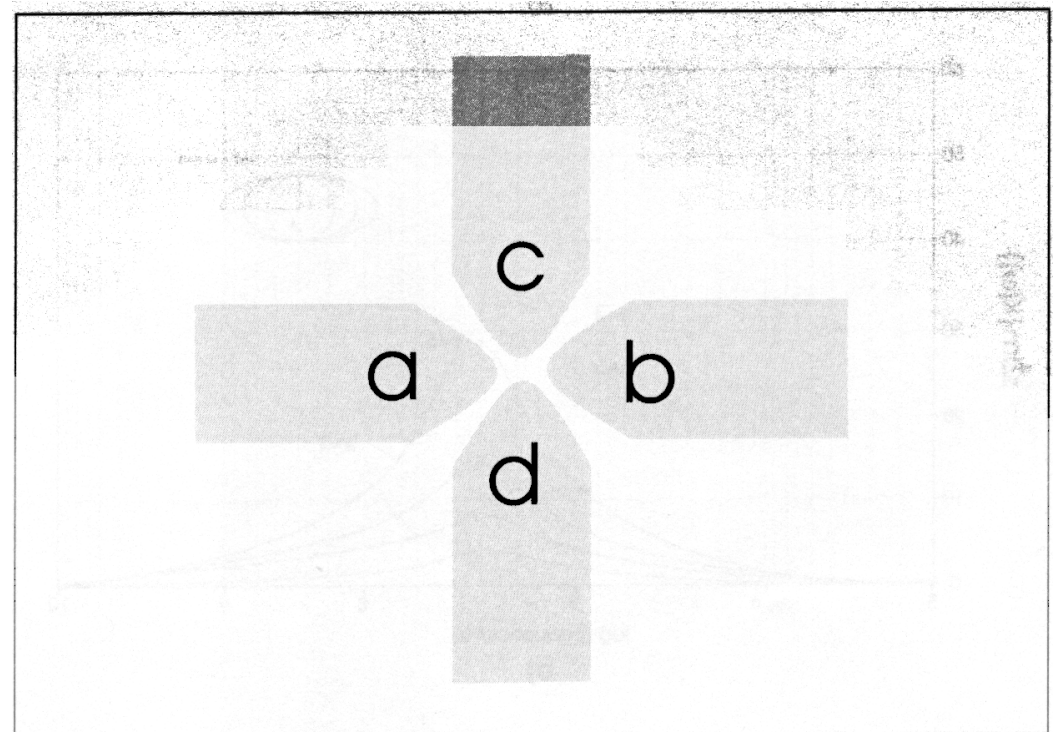

Figure 6. Typical quadrupole electrode microstructure. ${ }^{3}$

${ }^{3}$ The gap between opposing electrodes in the centre of the array may be as small as $500 \mathrm{~nm}$ or as large as $1 \mu \mathrm{m}$. Such a structure can be used for both dielectrophoresis and electrorotation, depending on how the electrodes are powered. If $\mathbf{a}$ and $\mathbf{b}$ are of the same phase, and $\mathrm{c}$ and $\mathrm{d}$ are in antiphase to them, dielectrophoretic motion is observed, while electrorotation of particles in the central part requires $\mathrm{c}, \mathrm{b}, \mathrm{d}$, phase-shiftet by $90^{\circ}, 180^{\circ}, 270^{\circ}$ whit respect to electrode a 


\section{APPLICATIONS TO NANOENGINEERING}

The separation of heterogeneous populations of normal and leukemic erythrocytes by dielectrophoresis was already shown as early as 1992 by Gascoyne et al [36]. Since then a broad variety of different cells including bacteria, yeast and cancer cells have been exposed to this technique [37-39]. Submicrometre populations of herpes simplex and tobacco mosaic virus were separated by Morgan et al [23].

Molecular sorting or filtering in a nanotechnological context by tuning the magnitude and frequency of the electric field can be suggested by the results, we show in Fig. 4. Cylindrical objects of different length suffer different dielectrophoretic forces in magnitude and direction, depending on the frequency of the electric field. Blocomputers based on DNA, in order to be read out, need exactly such kind of sorting.

Traveling Wave dielectrophoresis devices could be used as 'conveyor belt' for 'lab-on-a-chip' or even 'factory-on-achip'- applications, wherein analyses of different chemical processes may be carried out on the same chip.

The concentration of particles from a colloidal solution in a defined space between the field gap of electrodes is an obvious nanotechnological application and has been shown many times, including to precipitate DNA and proteins [40]. Microscopic biosensors by stacking particles of different types, which were driven by dielectrophoretic force to contact and bind, have been constructed by velev et al [41].

Electrorotation of nanoparticles has the potential as means of inducing rotation in a molecular machinery. Indeed Berry et al [42] have shown that the molecular motor of $E$. coli bacteria could be driven backwards with rotation frequencles of up to $2 \mathrm{kHz}$. The induction of torque in micro-scale motors by electrorotation has been achieved by Hagedorn et al [43].

\section{CONCLUSIONS}

One of the principal requirements for the advancement of nanoengineering and molecular electronics is the development of precision tools for the manipulation of macromolecules and biological cells in solution phase. A.c. electrokinetic effects, as described in this paper, are produced with arrays of electrodes, which on a nanometer scale represent this kind of tools for trapping, manipulation and separation of particle populations and finally for maneuvering smaller single particles and molecules

\section{ACKNOWLEDGEMENT}

Financlal support of the BUAP through grant III 35 G02 (2003) is kindly acknowledged

\section{RFFERFNCFS}

11 Drexler KE., Molecular Engineering: an Approach to the Development of General Capabilities for Molecular Manipulation, Proc. Natl. Acad. Sci. USA, Vol. 78, N0. 9, pp. 5275-5278, 1981.

Polla L., Erdman G., Robbins P., Markus T., Diaz-Dlaz, Nam, and Brickner, Wang and Krulevitch, Microdevices in Medicine, Annu. Rev. Biomed. Eng. 02:551-76, 2000.

[3] Adleman LM., Molecular computation of solutions to combinatorial problems, Science 226, 1021-1024, 1994.

[4] Drexler KE., Nanosystems; Molecular Machinery, Manufacturing and Computation, New York: Wiley, 1992.

[5] Feynman R., Infinitesimal machinery, reprinted in J. Micromech. Syst. 2, 4-14, 1993.

[6] Sitti M., Survey of Nanomanipulation Systems, Proc. of the IEEE-Nanotechnology Conference, pp. 75-80, 2001.

[7] Binnig G., Quate C.F., and Gerber C., Atomic Force Microscope, Phys. Rev. Lett., Vol. 56, pp. 930-933, 1986.

[8] Hillner P., Walters D., and et al., Combined atomic force and confocal laser scanning microscope, JMSA, Vol. 1 , pp. 127-130, 1995.

[9] Green NG., Morgan $H_{\text {., }}$ and Milner $\Perp$., Manipulation and trapping of sub-micron bioparticles using dielectrophoresis, J. Biochem. Biophys. Methods, 35, 89-102, 1997. 
Batchelder JS., Dielectrophoretic manipulator, Rev. Sci. Instrum. 54, 300-2, 1983. Zehe A., Tecnología Epitaxial de Silicio, pp 1-305, ISBN 3-8311-1438-2 Editorial intercon (Alemania, Norderstedt) 2000.

[1 2] Zehe A., Moletrónica: La electrónica a escala molecular entre semiconductores y arreglos moleculares, http://unw.moletronica.buap.mx

Chuart T., \& Deschenaux, Desing R., Mesomorphic properties, and supramolecular organization of $\left(C_{60}\right)$ fullerence- containning thermotroi cliquid crystal. J. Mater. Chem. 12, 1944-1951, 2002.

[141 Braach-Makswytis V., Raguse B., Nanobiosystems, APEC Meeting on Nanotechnology, Nov. 5-7, Ottawa, Canada, 2001.

¡151 Herman MA., Sitter H., Molecular Beam Epitaxy, Springer- Verlag Berlin Heidèlberg New York, London, Paris, Tokyo, 1989.

[1 161 Christensen AN., (ed.), Crystal Growth and Characterization of Advanced Materials, World Scientific Publishing Co. Pte. Ltd., ISBN 9971-50-730-7, 1988.

[17] Roberts GG., ed., Langmuir-Blodgett Films: An Introducción, Cambridge, Great Britaln: Cambridge University Press, 1996.

[18] Ulman A., An Introduction of Ultrathin Organic Films., New York: Academic Press, 1991. Jones TB., Electromechanics of Particles Cambridge: Cambridge University Press, 1995.

[วn1 Ramos A., Morgan H., Green NG., and Castellanos A., AC electrokinetics: a review of forces in microelectrode structures, J. Phys. D: Appl. Phys. 31 2338-53, 1998.

[211 Zimmermann U., and Neil GA., Electromanipulation of Cells, Boca Raton, F.L.: Chemical Rubber Company, 1996.

[1)1 Washizu M., Suzukj S., Kurosawa O., Nishizaka T., and Shinohara T., Molecular dielectroforesis of biopolymers, IEEE Trans. Ind. Appl. 30 835-42, 1999.

[7 31 Morgan H., Hughes MP., and Green NG., Separation of sub-micron particles by dielectrophoresis, Blophys. J. 77 516-25, 1999.

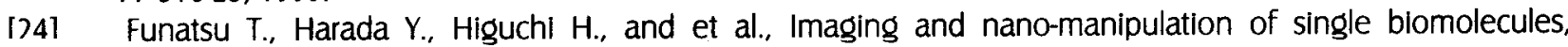
Biophysical Chemistry, Vol. 68, pp. 63-72, 1997.

[751 Katsura S., Yamaguchi A., and et al., Manipulation of globular DNA molecules for sizing and separation, Electrophoresis, Vol. 21, pp. 171-175, 2000.

[D61 Yamamoto T., Kurosawa O., Molecular surgery of DNA based on electrostatic micromanipulation, IEEE Trans. on Industry Appl., Vol. 36, no. 4, pp. 1010-1017, 2000.

1771 Mehta A., Rief M., and et al., Single-molecule biomechanics with optical methods, Science, Vol. 283, pp. 16891695, 1999.

[281 Ishii Y., Ishijima A., and Yamagida T., Single molecule nanomanipulation of biomolecules, Trends in Biotechnology, vol. 19, no. 6, 2001.

[291 Landau LD., Lifschitz EM., Elecktrodymanik der Kontinua (Continuum Electrodynamics), Vol. 8 Akademic-Verlaø. Berlin, 1985.

[30] Bones TB., Electromechanics of Particles, Cambridge Univ. Press, Cambridge, 1995.

[31] Paul R., Otwinowskj M., J. Theor. Biol. 148 495, 1991.

[32] Bohren CF., Huffman DR., Absorption and Scattering of Light by Small Particles, Wiley, New York, 1983.

[33] Sebastián JL., Muñoz S., Sancho M., Miranda J., Phys. Med. Biol. 46 213, 2001.

[341 Hughes MP., Computer-aided analysis of conditions for optimizing practical electrorotation, Phys. Med. Biol. 12, 3639-48, 1998.

I351 Reichle, Müller, Schnelle and Fuhr, Electro-rotation in octopole micro cages, J. Phys. D: Appl. Phys. 32, 21282135, 1999.

[361 Gascoyne PRC., Huang Y., Pethig R., Vykoukal J., and Beccker FF., Dielectrophoretic separation of mammalian cells studied by computerised image analysis Meas. Sci. Technol. 3, 439-45, 1992.

[371 Markx GH. and Pething R., Dielectrophoretic separation of cells: continuous separation, Biotechnol. Bioeng. 45, 337-43, 1994.

[38] Pethig R., Dielectrophoresis: using inhomogeneous AC electrical fields to separate and manipulate cells, Crit. Rev. Biotechnol. 16. 331-48. 1996. 
[39] Hughes MP., Morgan H., Rixon F]., Burt JPH., and Pethig R., Manipulation of herpes simplex virus type 1 by dielectrophoresis, Biochim, Biophys. Acta 1425, 119-26, 1998.

[40] Washizu M., Kurosawa O., Arai I., Suzuki S., and Shimamato N., Applications of electrostatic strech and positioning of DNA, IEEE Trans. Ind. Appl. 31, 447-56, 1994.

[41] Velev O., and Kaler EW., In situ assembly of colloidal particles into miniaturized biosensors, Langmuir 15, 3693-2698, 1999.

[42] Zehe A., Ramírez A., The depolarization field in polarizable objets of general shape, Rev. Mex. Fís. 48, 427-431, 2002.

[43] Hagedorn R., Fuhr G., Müller T., Schnelle T., Schakenberg U., and Eagner B., Desing of asynchronous dielectric micromotors, J. Electrost. 33, 159-85, 1994. 